Deutsche Automobilhersteller und der Partikelfilter

\section{Ankunft in der Vergangenheit}

\section{Deutsche Automobilhersteller haben auf der diesjährigen Internationalen Auto- mobilausstellung die Einführung von Partikelfiltern bei einigen ihrer Diesel- PKW Modelle angekündigt. Dass die Autoindustrie jahrelang gegen den Filter gekämpft hat und in der Entwicklung der Technologie vier Jahre hinten dran ist, ging im großen Öffentlichkeitsrummel unter. Durch die lange Abwehrhaltung hat die Industrie nicht die Reduktion von schädlichen Partikeln verzögert, sondern auch ihre geschäftlichen Aussichten in den Dieselmärkten der Zukunft geschmälert.}

$\mathrm{F}$ Von Rüdiger Haum Feinstpartikel in den Abgasen von Dieselfahrzeugen sind gesundheitsschädlich. Zwar ist das exakte Ausmaß möglichen Schadens unklar, dass Menschen aber besser und länger ohne Partikel in die Lunge leben gilt als Konsens. Laut einer Studie des Umweltbundesamtes verursachen Dieselemissionen zwischen 10.000 und 18.000 Krebserkrankungen im Jahr (1). Seitdem die Schädlichkeit der durch unvollständige Kraftstoffverbrennung entstehenden Partikel bekannt ist, gibt es Streit um die sinnvolle Konzentration von Partikeln in den Autoabgasen. Umweltschützer, Politiker, Wissenschaftler und selbstverständlich auch die Automobilkonzerne zanken sich über die Höhe und die Erfüllungszeiträume der entsprechenden Grenzwerte.

Auf der diesjährigen Internationalen Automobilausstellung (IAA) hat die deutsche Automobilindustrie den Einsatz von Partikelfiltern vor in Kraft treten der nächsten Abgasnorm Euro 4 im Jahr 2005 angekündigt. Ein selbstloser, so mag man glauben, heroischer Akt zum Schutz von Umwelt und Gesundheit. Tatsächlich zelebrierte die Industrie den winzigen Schlusspunkt eines langwierigen Prozesses in dem weit mehr Akteure für Entwicklung und Einsatz des Dieselfilters bei PKWs verantwortlich sind (2). Die Autohersteller waren dabei eher hinderlich.

Die Regulierung von Schadstoffemissionen bei Fahrzeugen ist ein Politikbereich, der neben ökologischen Zielen stark von ökonomischen Interessen durchdrungen ist. Grundsätzlich schließen sich ökonomische und umweltpolitische Ziele aber nicht aus. Prinzipiell kommen Schadstoffreduktionen von Automobilen durch politische Verordnung von Grenzwerten und deren Einhaltung durch technologische Weiter- entwicklungen der Fahrzeuge zu Stande. Die Diffusion der Technologien ist allerdings kein simples Wechselspiel von politischer Vorlage und Reaktion der Automobilhersteller. Es ist ein komplexer Prozess, der durch eine Vielzahl von Faktoren sowie Akteuren bedingt und gestaltet wird.

\section{Industrie blockiert in Brüssel}

Im Fall des Partikelfilters begann der Streit um Grenzwerte, bei dem die Automobilindustrie sich vehement gegen zu viel umweltfreundliche Technik wehrte, 1994. Im Rahmen des Forschungsprogramms Auto-Öl 1, an dem sich EUKommission sowie die Verbände der Automobilhersteller und Ölindustrie beteiligen, wurde über die momentan gültige Abgasnorm Euro 3 und die ab 2005 gültige Abgasnorm Euro 4 verhandelt. Der 1996 veröffentlichte Entwurf für eine entsprechende Richtlinie für neue PartikelObergrenzen wurde sowohl von NGOs als auch vom Europäischen Parlament als zu lax und, entgegen den Aussagen der Industrie, mit wenig technologischem Aufwand einhaltbar kritisiert. Die Kritiker betonten, dass beide Industrieverbände ihre Stellung innerhalb des Programms nutzten, um durch überhöhte Kostenschätzungen nur leichte Senkungen der Grenzwerte zu erreichen. Die 1998 schließlich beschlossenen Schadstoffgrenzwerte stellten nach schwierigen Verhandlungen zwischen Kommission, Rat und Parlament unter starkem Einfluss der Industrie lediglich einen Kompromiss dar. Um überhaupt eine Einigung zu erzielen, erforderten die ab 2000 einzuhaltenden Grenzwerte für Partikelmasse bei Diesel-Pkw fast gar keinen technologischen Entwicklungsaufwand seitens der Automobilhersteller. Als Ausgleich da- für waren die ab 2005 gültigen Grenzwerte strikter und verlangen die Entwicklung neuer Technologien zur Partikelreduktion. Das Jahr 2005 wurde gewählt, weil nach Industrieangaben die für die strikten Grenzwerte notwendigen Technologien frühestens zu diesem Zeitpunkt markreif seien. Die Einwände von technischen Sachverständigen bleiben weitgehend ungehört. Nach Schätzungen des Umweltbundesamts von 1995 hätte beispielsweise der Partikelfilter, der fast 100 Prozent der gesundheitsschädlichen Partikel aus den Abgasen herausnimmt, bereits 1998 auf dem Markt sein können.

Gut ein Jahr später schon ist die Zeitschätzungen der Automobilhersteller überholt. Der französische Hersteller Peugeot kommt mit einem Partikelfilter für einen Teil seiner PKW-Modelle auf den Markt, 2000 auch in Deutschland. Peugeot demonstriert die technische Machbarkeit des Partikelfilters und gibt all jenen recht, die die Entwicklungszeit kürzer geschätzt haben. Das Motiv von Peugeot ist strategisch. Die Konzernleitung erhofft sich Wettbewerbsvorteile bei umweltbewussten Autofahrern, Deutschland gilt als Testmarkt. Wenn der Filter hier ankommt, so sind die Chancen für eine weitere Verbreitung in Europa gut. Die deutsche Automobilindustrie hatte zur Einhaltung der in Euro 4 festgelegten Schadstoffgrenzen auf die Entwicklung von innermotorischen Maßnahmen gesetzt. Bei innermotorischen Maßnahmen entstehen durch Verbrennungsoptimierung weniger Rußpartikel, es verschwinden aber längst nicht alle Partikel aus den Abgasen. Innermotorische Maßnahmen sind außerdem nur bei kleineren Motormodellen zur Einhaltung von Euro 4 ausreichend. Für größere Modelle wären auch die deutschen Automobilhersteller nicht um den Einsatz von Partikelfiltern herumgekommen. Eine Einführung vor 2005 allerdings nicht geplant.

\section{Öffentlicher Zweifel}

Als Reaktion darauf versuchten die deutschen Automobilherstellern den Partikelfilter von Peugeot mit Hinweisen auf technische Mängel zu diskreditieren. Die Dauerhaltbarkeit des Filters sei nicht gewährleistet und der Kraftstoffverbrauch durch den Filtereinsatz erhöht. Der Vorschlag des Bundesumweltministers, auf freiwilliger Basis Partikelfiltersysteme einzuführen, wurde abgelehnt. Zusätzlich bestritt der Verband der deutschen Automobilindustrie die Existenz serienmäßig einsetzbarer Partikelfilter (3). Auch die zu diesem Zeitpunkt in der Öffentlichkeit wieder 
stärker aufkommende Diskussion um die Gesundheitsgefährdung der Partikel konnte der Position der deutschen Hersteller nichts ändern.

Erst im Sommer 2001 waren die deutschen $\mathrm{Au}$ tomobilhersteller gezwungen, die technische Kritik am Filter einzustellen. ADAC und Umweltbundesamt (UBA) bewiesen durch einen Langzeittest, dass der Filter auch bei dauerhafter Belastung einwandfrei funktioniert und kein signifikanter Spritverbrauch festzustellen ist. Durch den Test von ADAC und UBA erhöhte sich der öffentliche Druck auf die Hersteller enorm. Umweltverbände und Medien forderten von den restlichen Herstellern, mit Peugeot gleichzuziehen. Deren Haltung änderte sich dadurch allerdings wenig. Sie lehnten den Filter weiter ab, änderten aber ihre offizielle Kommunikationsstrategie. Der Zweifel an der Funktionsfähigkeit wich in Pressemitteilungen und Stellungnahmen des Jahres 2001 Hinweisen darauf, dass die Euro-4-Norm keine Vorgaben in Bezug auf die einzusetzende Technologie mache. In dieser Hinsicht sei der Partikelfilter von Peugeot nichts weiter als ein Marketing-Gag. Ihrer Umweltverantwortung seien sie durch die vorzeitige Einhaltung der Norm bei einigen Modellen durch verbesserte innermotorische Verbrennung gerecht geworden.

Ein Strategiewechsel, obwohl zeitlich schwer einzugrenzen, ergab sich erst im Laufe des Jahres 2002. Dies mag auch mit der im November 2002 begonnenen, zivilgesellschaftlichen Initiative „Kein Diesel ohne Filter“ zu tun haben. Ihr Ziel ist, eine steuerliche Förderung des Partikelfilters durch Öffentlichkeitsarbeit und Lobbying durchzusetzen. Im Frühjahr 2003 mehrten sich plötzlich die Hinweise, dass weitere Hersteller den Dieselfilter vor 2005 einzuführen planten. Öffentlich wurde aber weiter geschimpft. Allen voran beklagte sich VW-Vorstandschef Pieschetsrieder über mangelnde Entwicklungsfreiheit und Würdigung der Leistungen der deutschen Automobilindustrie in Sachen Umweltschutz. Anstatt bei den technischen Strategien der Hersteller hineinzufunken, solle lieber die Gesetzgebung auf europäischer Ebene voran getrieben werden.

\section{Fazit}

Die auf der IAA angekündigte Einführung des Partikelfilters ist nicht der alleiniger Verdienst der deutschen Hersteller. Wäre es nach ihnen gegangen, wäre der Filter erst 2005 auf dem Markt. Erst ADAC, UBA und einer Reiher ande-

rer ziviler Akteure sowie die Marketingstrategien von Peugeot haben die deutschen Hersteller zu dem gezwungen, was sie Ende der neunziger Jahre in Brüssel so lange wie möglich haben herauszuzögern versucht. Durch die Verzögerung muss nun doppelter Schaden konstatiert werden. Erstens hat sich die Reduktion beim Partikelausstoß unnötig verzögert. Zweitens sind entscheidende technische Fortschritte nun bei anderen Herstellern gemacht wurden. Langfristig gesehen werden die Grenzwerte für Partikel weltweit schärfer werden. Um in den DieselMärkten der Zukunft, beispielsweise den USA, überhaupt mitzuspielen ist Know-How über Partikelfilter unverzichtbar. Den Wissensvorsprung besitzt nun Peugeot und die verbundenen $\mathrm{Zu}$ lieferer, die bereits vier Jahre Erfahrung und einen Marktvorteil mit der Technologie haben. VW und Co gucken trotz aller Entwicklungsbemühungen vorerst in die Röhre.

\section{Anmerkungen}

(1) Wichmann, Erich: Abschützung positiver gesundheitlicher Wirkungen durch den Einsatz des Partikelfilters bei Dieselfahrzeugen in Deutschland. Gutachten im Auftrag des Umweltbundesamtes, Berlin 2003.

(2) Der Artikel beruht auf einer Fallstudie, die im Rahmen des BMBF-Verbundprojektes „LEAD-MARKET: Joint project Policy-Frameworks for the Development of International Markets for Innovations of a Sustainable Economy - from Pilot Markets to Lead Markets" erstellt wurde. Vgl.: Rüdiger Haum/ Ulrich Petschow: Lead markets for environmental technologies: The case of the particulate filter for Diesel passenger cars. IÖW Diskussionspapier 59/03, Berlin 2003. Download: www.ioew.de

(3) Vgl.: VDA: Jahresbericht Verband der Automobilindustrie e.V. Frankfurt am Main 2000.

\section{Der Autor}

Rüdiger Haum ist wissenschaftlicher Mitarbeiter im Forschungsfeld Umweltpolitik und Umweltökonomie am Institut für ökologische Wirtschaftsforschung (IÖW).

Kontakt: IÖW, Potsdamer 105, 10785 Berlin. Tel. 030-8845940, E-Mail: Ruediger.Haum@ioew.de
Anzeige 
(c) 20I0 Authors; licensee IÖW and oekom verlag. This is an article distributed under the terms of the Creative Commons Attribution Non-Commercial No Derivates License (http://creativecommons.org/licenses/by-nc-nd/3.o/), which permits unrestricted use, distribution, and reproduction in any medium, provided the original work is properly cited. 